\title{
The effect of tube end constraining on the axial crushing behavior of an aluminum tube
}

\author{
Alper Tasdemirci * \\ Department of Mechanical Engineering, Izmir Institute of Technology, Cevre AR-GE Binasi, Gülbahçe Köyü, Urla, 35430 Izmir, Turkey
}

\section{A R T I C L E I N F O}

\section{Article history:}

Received 12 December 2007

Accepted 3 April 2008

Available online 11 April 2008

\section{Keywords:}

E. Mechanical

F. Plastic behavior

G. Destructive testing

\begin{abstract}
A B S T R A C T
The effect of various types of end constraining on the deformation and load-displacement behavior of a 3003-H14 Al tube were experimentally and numerically studied. No effect of single-end constraining of tubes was found. Few conditions of double-end constraining tended to revert the deformation mode to mixed and/or diamond mode of deformation. Double-end constraining of tube ends further resulted in an increase in initial drop-load values, widening the initial overshot region in average load-displacement curves. The agreement between numerical and experimental results showed the capabilities of the used numerical model in order to predict end-condition effects in tubular structures.
\end{abstract}

(c) 2008 Elsevier Ltd. All rights reserved.

\section{Introduction}

Tubular structures are widely used as crush energy mitigating elements in various kinds of structural applications. The choice of tubular structures, including circular and square tubes, for energy absorption applications lies in the fact that they progressively deform under compressive loads through one of the plastic folding mechanisms of concertina, diamond and mixed mode, resulting in deformation energy absorption at a nearly constant load. An analytical model for predicting the average crushing loads of progressive axisymmetric folding of thin-walled tubes was first shown by Alexander in 1960 [1]. The model has been extended by including the curvature of fold [2,3], internal folding [4] and both internal and external folding [5]. The average crushing load of the progressive asymmetric folding was investigated by Pugsley and Macaulay [6], Abramowicz and Jones [2] and Singace [7]. Generally, tubes with low $D / t$ ratios and rigidly perfectly plastic materials exhibit axisymmetric mode of deformation, while tubes with high $D / t$ ratios and strain hardening materials exhibit asymmetric mode of deformation [8]. Mixed mode is generally observed following the first couple of axisymmetric mode of folding. The concertina mode of deformation of HT30 Al alloy tubes was found to be prevalent, when $D / t$ ratios varied between 10 and 50 and $L / D$ ratios between 1 and 5 [9]. Due to the increased fold length and the promotion of the global bending, diamond mode of deformation is usually regarded as the lower mode of deformation as compared with concertina mode. In addition, the experimental deformation and load-

\footnotetext{
* Tel.: +90 232 7507817; fax: +90 2327507890 .

E-mail address: alpertasdemirci@iyte.edu.tr
}

displacement curves of tubes were affected by several other factors including the folding parameter (ratio of internal folding to fold length) [5], load eccentricities [10], cut-off and grooving on the tube wall [11,12], foam-filling [13-15] and any disturbances in the periodicity of folding. In aforementioned earlier studies, the effect of end constraining was generally ignored; the tube ends deformed freely. The effect of end-condition on the deformation and load-displacement curves of $\mathrm{Al}$ and steel tubes was previously studied by Singace and El-Sobky [8]. It was shown that the singleend inward constraining of short Al tubes resulted in a change of deformation mode from concertina to diamond, while double constraint tubes showed separated ring mode of deformation. Moreover, in double-end constraint tubes, the initial overshoot in the load-displacement curve, which is responsible for the overestimation of average crushing load, was removed. The effects of side constraints on the deformation and load-displacement curves of hexagonal and square packed tubes were investigated by Guden and Kavi [16]. It was shown that the side constraining of the tube bundles increased the average crushing loads over those of single tubes. These previous studies showed that the empty tube deformation modes were affected by the tube material type, heat treatment applied, geometrical parameters and end constraint.

In parallel with the experimental studies there has been a growing interest in numerical simulations of the axial crushing of the tubular structures. Numerical simulations have capabilities in showing the initiation and propagation of the axial crushing and the effects of various parameters on the energy absorption characteristics. In addition, recent advancements in finite element analysis software and computation technology have been allowing researchers to successfully simulate the axial crushing of tubular structures in an acceptable period of time. Once the model 
becomes well-verified, i.e. successfully capture the experimental data, then, the parametric studies can be done at a fraction of the cost of the experimental studies. Aljawi [17] studied the axial crushing of circular tubes experimentally and numerically and found well agreement between the numerical force histories of tubes with those obtained by the experiments. Bardi et al. [18] studied the axisymmetric progressive crushing of circular tubes, both experimentally and numerically. The used model demonstrated that the changes in the loading cycles with increasing the number of folds were due to the differences between the inner and outer folds, which in turn affected the self contact of the fold walls. Al Galib and Limam [19] studied experimentally and numerically the quasi-static and dynamic crushing behavior of circular aluminum tubes. The numerically predicted crushing forces and the fold formation showed well agreements with those of the experiments. Some further observations were also made on the effects of geometrical imperfections and deformation rate on the crushing behavior of circular tubes. Silcock et al. [20] used the explicit code LS-DYNA to simulate the crushing behavior of steel tubes. It was found that the numerical crush modes, load-deflection characteristics and energy absorption values showed reasonably good correlations with those of experiments. Previous numerical studies were quite successful in predicting the deformation modes of free-constraint empty tubes and can be extended to model the crushing behavior of constraint empty tubes.

The aim of the present study is to determine, experimentally and numerically, the effects of various end-conditions on the deformation mode and load-displacement and average load-displacement curves of short Al tubes deforming in concertina mode of deformation in free-constraint condition. The consistency between experimental and numerical results revealed that the numerical methodology used in this study could be further used to predict the deformation mode and load-displacement curves of the geometries with complex end-constraint types.

\section{Experiments and modeling}

\subsection{Materials and testing}

Aluminum deep drawn tubes (3003-H14) of $20 \mathrm{~mm}$ in outside diameter, $50 \mathrm{~mm}$ in length and $0.9 \mathrm{~mm}$ in wall thickness were subjected to quasi-static axial compression test at a cross-head speed of $100 \mathrm{~mm} \mathrm{~min}^{-1}$ using a displacement controlled SHIMADZU AG-I universal test machine with free-, single- and double-end constraints similar to those studied by Singace and El-Sobky [8]. The schematics of the constraint tubes are sequentially shown in Fig. 1 and the coding used in this figure is as follows: (1) free-end constraint, (2) single-end inward constraint, (3) single-end outward constraint, (4) single-end fully constraint, (5) double-end inward constraint, (6) double-end outward constraint, (7) double-end fully constraint, (8) double-end inward-outward constraint, (9) double-end inward-fully constraint and (10) double-end outward-fully constraint. The constraining end-caps were machined from a cold rolled steel bar with high tolerances. Fig. 2a-c show the machined inward, outward and fully constraint end-caps sequentially. The tubes were constrained at the ends with a section length of $3 \mathrm{~mm}$. Since the length of the tubes was chosen $50 \mathrm{~mm}$, the deformed lengths of the single and double-end constraint tubes were 47 and $44 \mathrm{~mm}$, respectively. For each configuration at least three tests were conducted. During the tests, the deformation of the tubes was video recorded and the records were compared with those of numerical models. The average crushing load $\left(P_{\mathrm{a}}\right)$ was calculated using following relation:

$P_{\mathrm{a}}=\frac{\int P \mathrm{~d} \delta}{\delta}$

where, $P$ and $\delta$ are the load and the displacement, respectively.

\subsection{Modeling}

The explicit non-linear finite element code LS-DYNA 971 was used to model the deformation of the circular tubes subjected to axial crushing with different end constraints. In order to correctly capture the deformation behavior of the tubes, a 3D full model was built. 3D and 2D views of the representative finite element model of a double-end fully constraint tube with the end-caps are shown in Fig. 3a and b, respectively. The tubes were modeled using Belytschko-Tsay shell element with
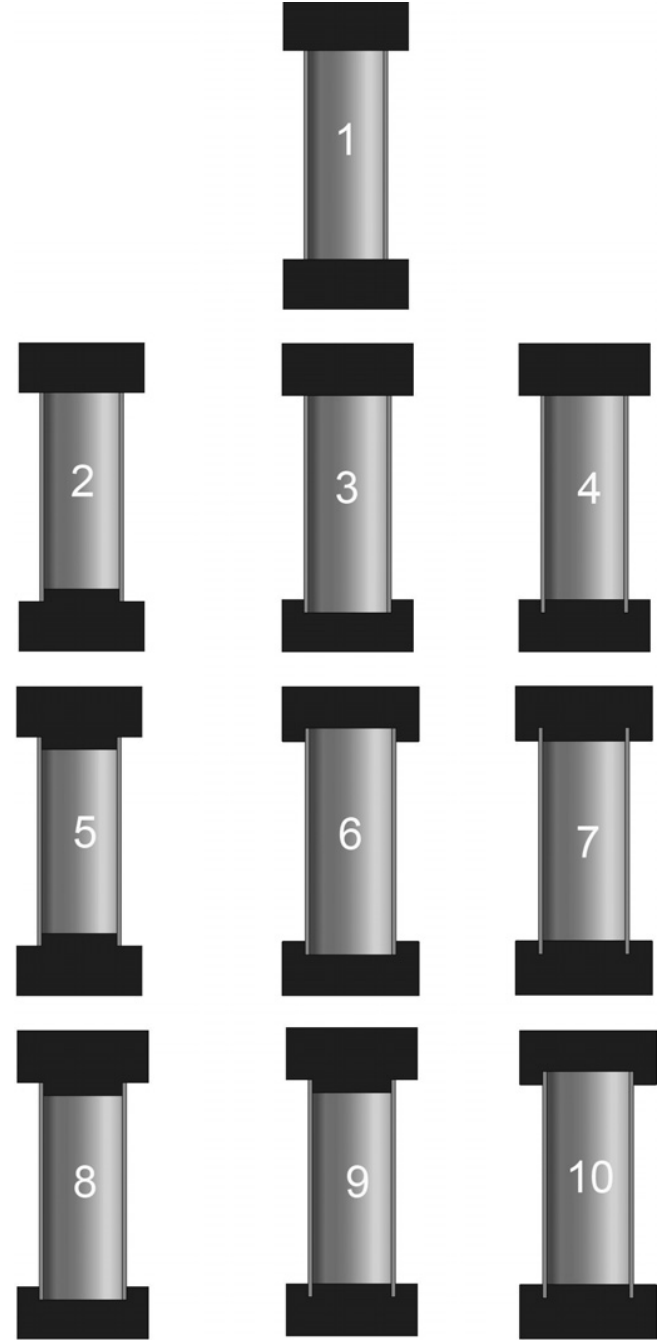

Fig. 1. Schematic of end-constraints: (1) free-constraint; (2, 3 and 4) single-end constraint; (5-10) double-end constraint.

nine integration points through the thickness. The optimum number of elements was determined by refining the mesh until the convergence reached. In order to check this effect the tube was meshed, for free-end constraint condition, with three different mesh densities: a coarse mesh with $25 \times 25$ elements, a medium sized mesh with $50 \times 50$ elements and a fine mesh with $75 \times 75$ elements through circumferential and axial directions. The load displacement curves are given in Fig. 3c. It was found that the medium sized mesh was able to give converged solutions within a reasonable amount of time thus this mesh size was used throughout the study. The load was applied to the upper end-cap modeled with solid elements. The rotational degrees of freedom were fixed at both ends of the tubes to avoid the unrealistic deformation modes. The displacements and rotations of the rigid endcaps were prevented, except the displacement of the upper end-cap along the vertical axis. The deformation rates were relatively small for an explicit solver; therefore, mass scaling was applied in order to achieve sufficiently small time steps and speed up the solution. As given in [14], the masses of the end-caps and the tube were scaled up while still maintaining a quasi-static deformation condition. The masses of end-caps and tube were scaled-up by a factor of 1000 . The ratio of the total kinetic energy (KE) to the total internal energy was found to be less than $4 \%$ over the period of the axial crushing, ensuring the quasi-static strain rates. The upper end-cap was displaced at a constant displacement rate of $100 \mathrm{~mm} \mathrm{~min}^{-1}$, same with that of experiments.

Two different types of contact were employed in the numerical simulations. For the tube itself, an automatic single surface contact was adopted to account for the contact between folds during deformation, while an automatic surface to surface contact was applied between the tube and rigid end- caps. A static friction coefficient of 0.3 and a dynamic friction coefficient of 0.2 between all surfaces in-contact were assumed in the simulations. The tube was modeled using an elasto-plastic material model (material model \# 24 in LS-DYNA) with isotropic hardening and von-Mises yield criterion. This material model allows for the input of an arbitrary stress-strain curve. The stress-strain behavior of the tube material shown in 


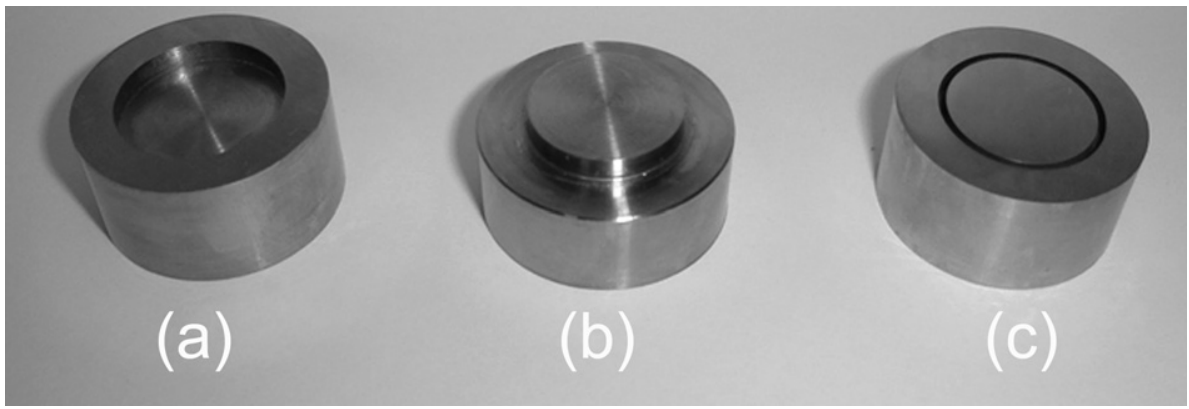

Fig. 2. End-caps: (a) inner, (b) outer and (c) fully constraints.

a

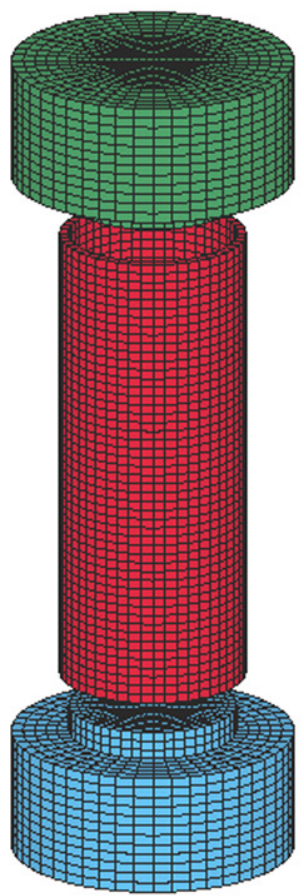

b
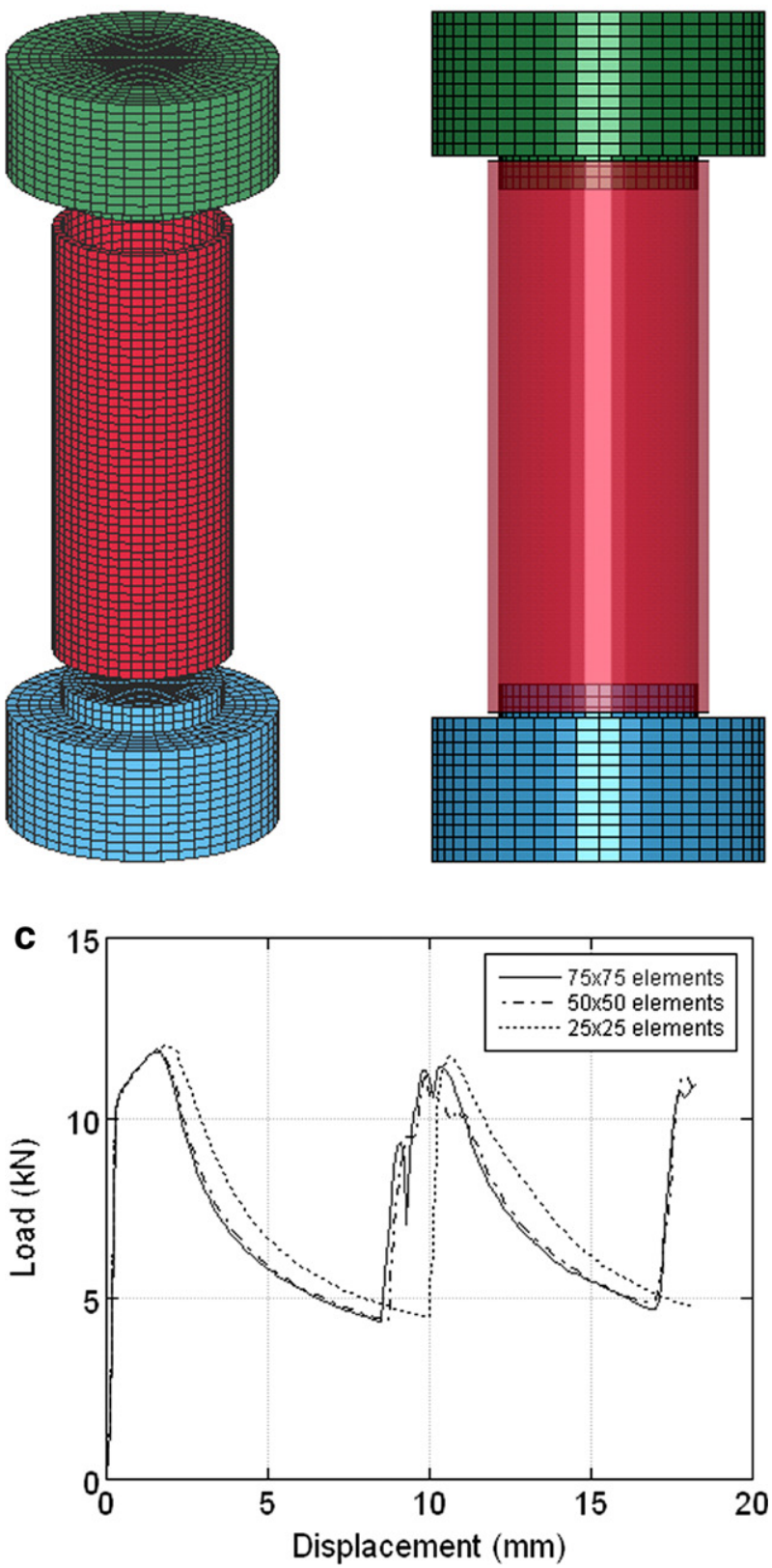

Fig. 3. Finite element models of a double-end fully constraint tube; (a) 3D and (b) 2D views. (c) Numerical load-displacement curves for different mesh sizes.

Fig. 4 was determined by the uniaxial tensile test (ASTM B557M) conducted at a cross-head speed of $100 \mathrm{~mm} \mathrm{~min}^{-1}$.

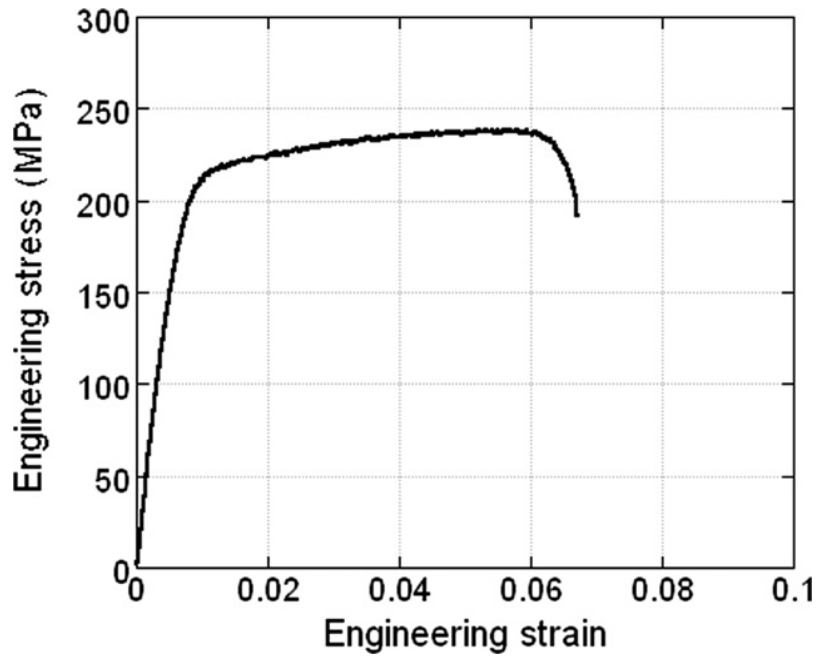

Fig. 4. Tensile engineering stress-strain curve of Al tube material.

\section{Results}

\subsection{Free-constraint tubes}

Free-constraint tubes deformed in concertina mode. In Fig. 5a, the load-displacement curves of three free-constraint tubes are shown together with the numerical load-displacement curve. Despite the relatively small variations in load values between each test, the experimental load-displacement curves show common features: a relatively high initial peak-load followed by a sharp decline in load values, and a gradual increase in the peak-load values following the initial peak-load as the displacement increases. Totally five lobes formed experimentally and numerically in the concertina mode of deformation. Further, the sequences of experimental and numerical concertina mode of deformation observed in this study were almost the same with those of previous studies $[9,18,19,21]$. The sequence of deformation is marked with the letters (A, B, C, D and E) and the numbers (1-10) in experimental and numerical load-displacement curves in Fig. 5a, respectively. Shortly, the deformation sequence consists of inward and outward bending of the tube wall. It was noted that initially, right before the appearance of the first peak-load (point A in Fig. 5a), two axisymmetric lobes were observed experimentally and numerically near the bottom and top ends of the tube. Following the maximum initial peak-load, one of the lobes continues to form: the tube wall bends outward and meanwhile the load values decrease until the point B in Fig. 5a; thereafter, the load values increase until the point $C$. During the inward folding of the tube wall over the first fold, the load values decrease once again (D in Fig. 5a). When 


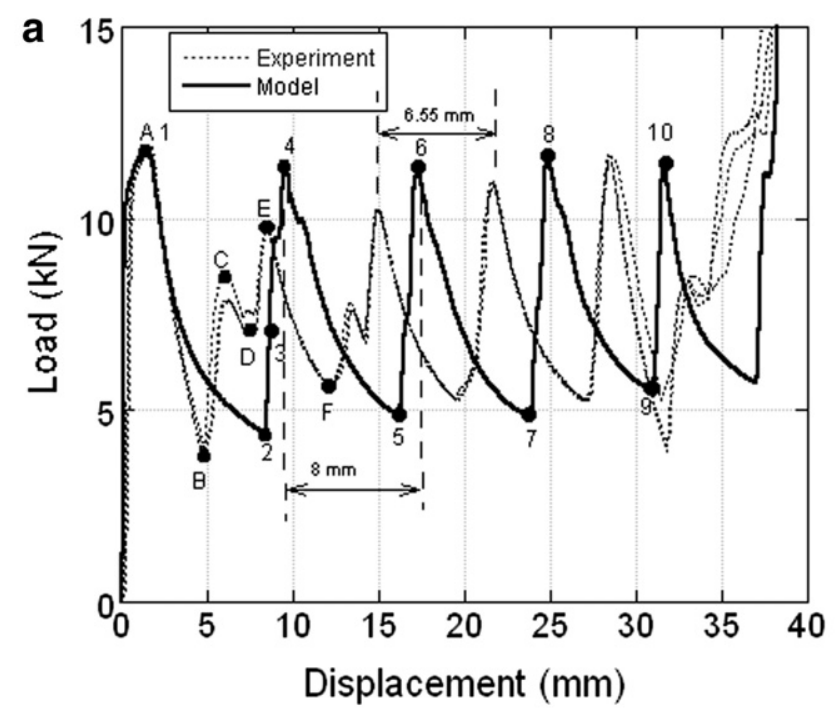

b

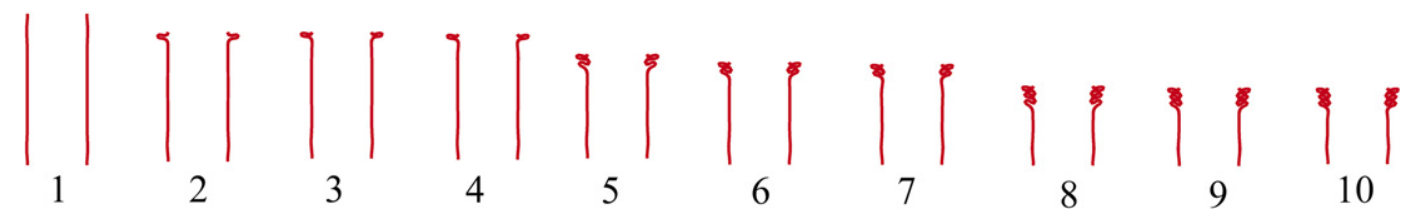

Fig. 5. (a) Experimental and numerical load-displacement curves and (b) the numerical deformation history of free-constraint tubes.

the inner wall of the first fold comes into contact, the load values increase until point $\mathrm{E}$, where outward folding starts to form over the first fold. When the outer knee of the second fold comes into contact with the first fold, the load values increase again from the point $\mathrm{F}$ in Fig. 5a. This sequence of tube wall deformation repeats as the third, fourth and fifth folds form, except the inner and outer fold formation (point $C$ and $D$, respectively) starts at the same point in the forth and fifth folds. The removal of the intermediate part of the cycle at the later stages of the tube folding is also noted previously [18]. The deformation sequence of the numerical tube crushing is also found to be very similar to that of experiments and also shown in Fig. $5 b$ at displacements corresponding to the numbers in Fig. 5b. Although the load values of the experimental and numerical load-displacement curves are very similar, the numerically calculated fold lengths are larger than those of experimental fold lengths. The fold length, for example, increases from $6.55 \mathrm{~mm}$ in experiments to $8 \mathrm{~mm}$ in numerical model in the initial deformation region as depicted in Fig. 5a.

\subsection{Single-end constraint tubes}

Single-end constraint tubes deformed, experimentally and numerically, in concertina mode of deformation as shown in Figs. 6 and 7, respectively. For comparison, the deformed picture of the free-constraint tube is also shown in both figures. In singleend constraint tubes, the folding always started from the free-constraint end and proceeded towards to the constraint end in both experimentally tested and numerical modeled tubes. Fig. 8a shows the load-displacement curves of single-end inward constraint tubes, together with that of free-constraint tube for comparison. Single-end and free-end constraint tubes essentially show very similar load-displacement behavior, confirming an insignificant effect of single-end constraining since the folding starts from the free-constraint end. Single-end outward constraint and singleend fully constraint tubes (not shown here) also showed very similar load displacement curves with that of free-constraint tube. As in the experimentally determined load-displacement curves, the

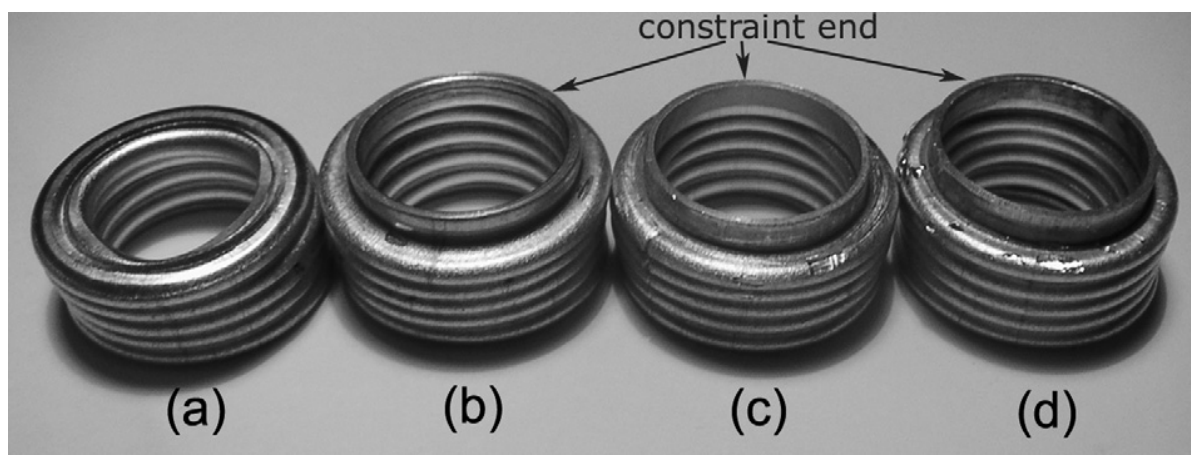

Fig. 6. Experimentally compressed (a) free and single-end constraint tubes, (b) inner, (c) outer and (d) fully constraint tubes. 


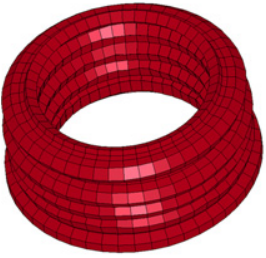

a

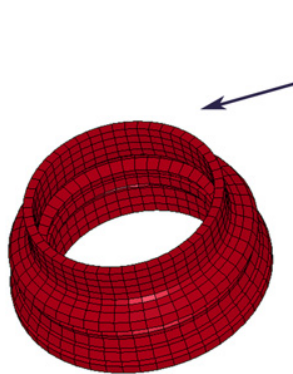

b constraint end

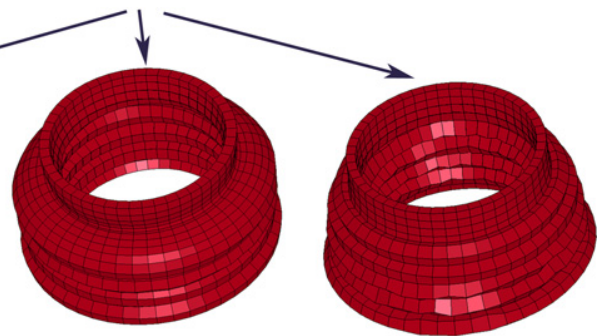

C

d

Fig. 7. Numerically compressed (a) free and single-end constraint tubes, (b) inner, (c) outer and (d) fully constraint tubes.
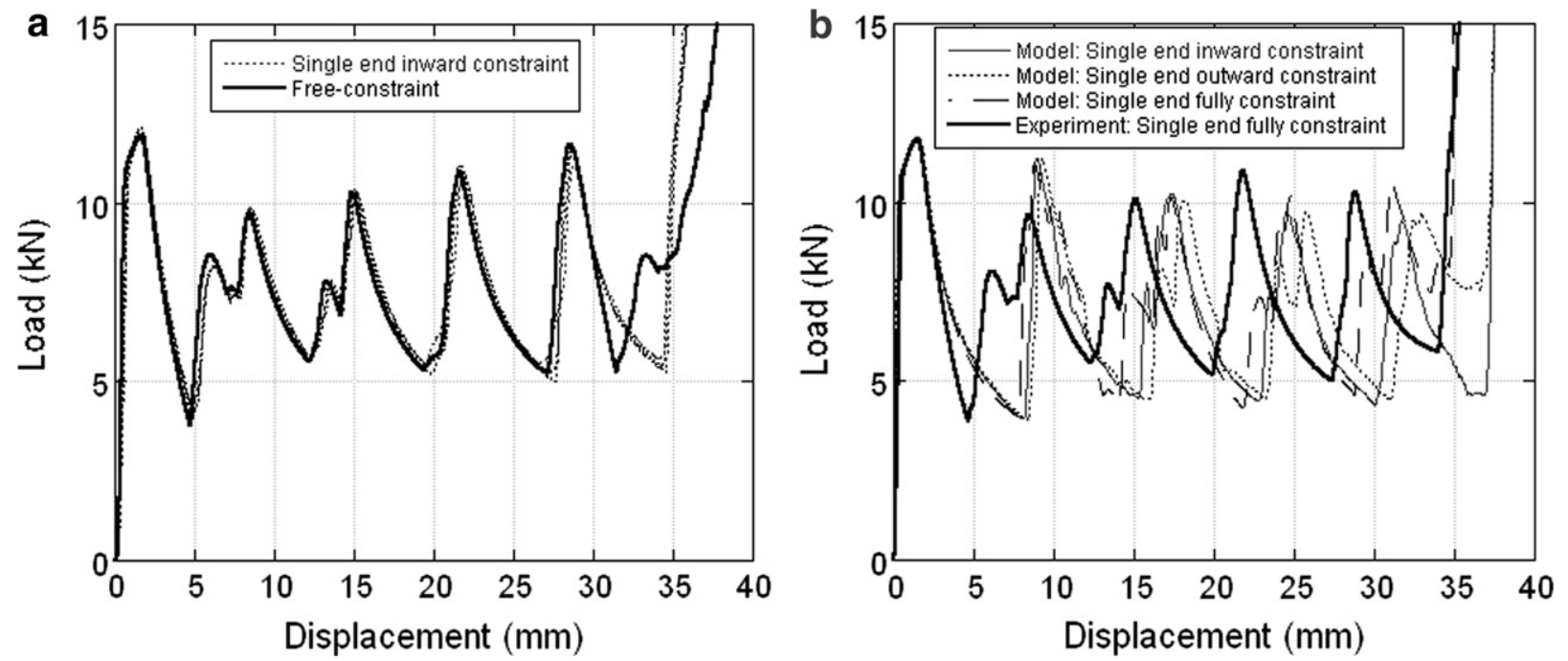

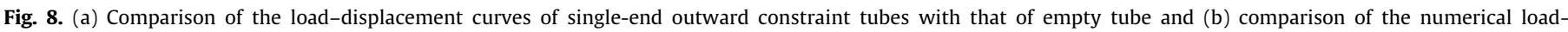
displacement curves with experiments.

numerical load-displacement curves of single-end constraint tubes are found to be very much similar (Fig. 8b). It is noted in Fig. 8b that, although initial peak-load values of the models and experiments are the same, experimental curves exhibit higher drop-load values than those of numerical curves. Despite the variations between the experimental and numerical load-displacement curves, the experimental and numerical numbers of folds (5) are the same for the single-end constraint tubes.

\subsection{Double-end constraint tubes}

In double-end inward constraint tubes, after the first axisymmetric fold, the deformation mode switches to asymmetric mixed/diamond mode as shown in Fig. 9a. The deformation mode of the double-end outward constraint tubes are concertina (Fig. 9b), while two double-end fully constraint tubes deformed in concertina mode (Fig. 9c) and one of them deformed in mixed mode (Fig. 9d). The numerical models for double-end inward and outward constraint tubes yield similar deformation modes as experimentally observed (Fig. 10a and b), while double-end fully constraint tube deforms in concertina mode of deformation as depicted in Fig. 10c.

Figs. 11a, 12a and 13 show sequentially load-displacement curves of double-end inward, outward and fully constraint tubes, together with the load-displacement curves of the corresponding models. As seen in these figures, the numerical load-displacement curves closely resemble the general trends of the experimental a

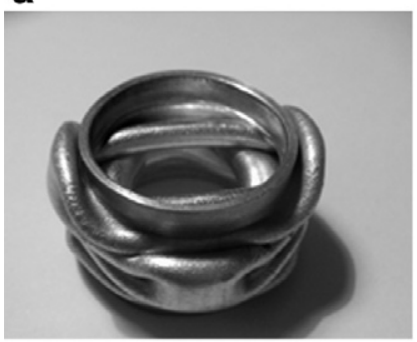

C

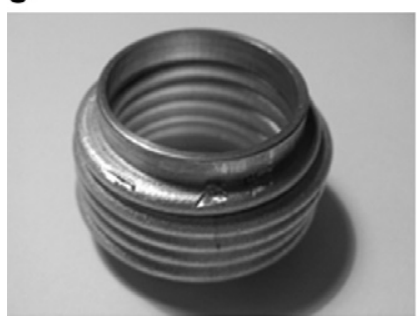

b

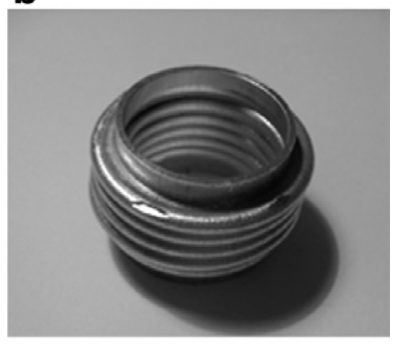

d

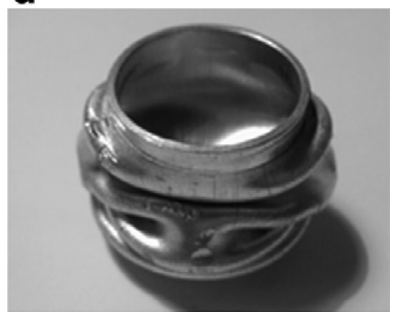

Fig. 9. Compressed double-end constraint tubes; (a) inward, (b) outward and (c and d) fully constraint.

load-displacement curves despite the relatively small discrepancies between the displacements corresponding to peak- and drop-loads. The effect of double-end inward constraint on the 


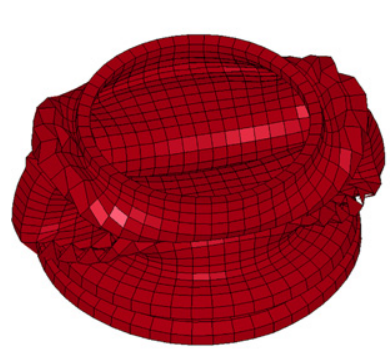

a

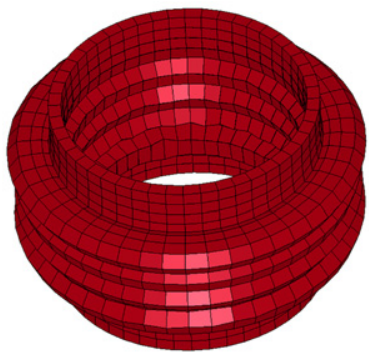

b

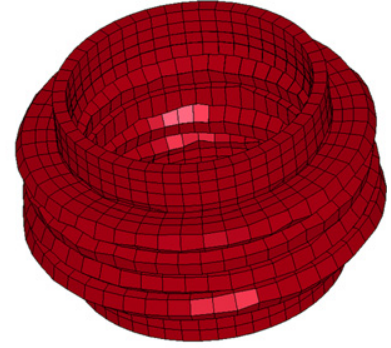

C

Fig. 10. Numerically compressed double-end constraint tubes (a) inward, (b) outward and (c) fully constraint.

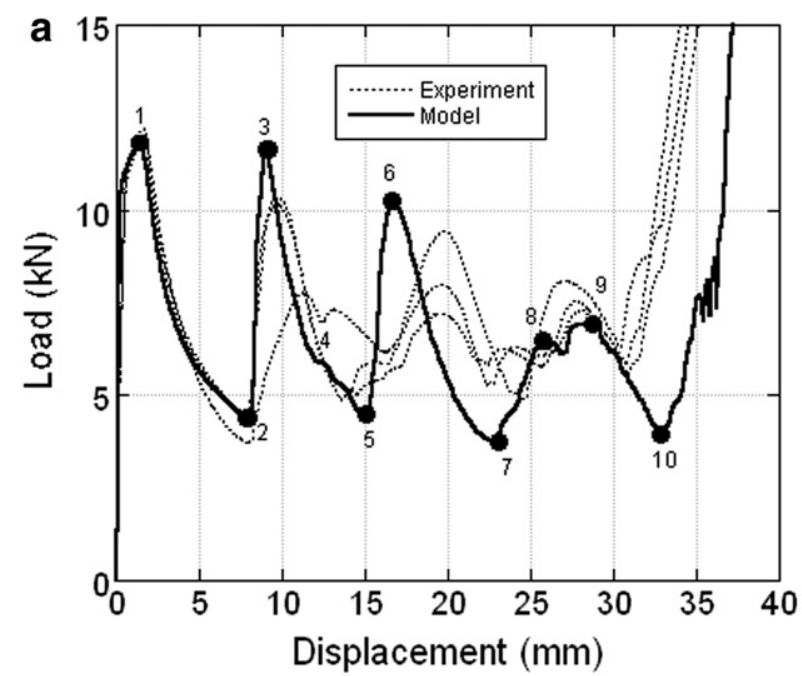

b

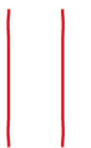

1

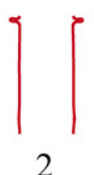

2

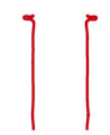

3

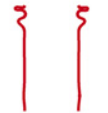

4

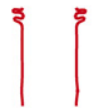

5

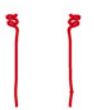

6

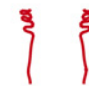

7

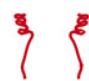

8

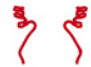

9

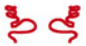

10

Fig. 11. (a) Experimental and numerical load-displacement curves and (b) numerical deformation history of double-end inward constraint tubes.

experimental load-displacement behavior of the tubes is to change the deformation mode from concertina to mixed/diamond mode. In the experiments, one axisymmetric fold is followed by three asymmetric folds in two tests and in the other, the tube crushes in diamond mode, while two axisymmetric folds are followed by two asymmetric folds in the model. The deformation sequence of the model is shown in Fig. 11b with the numbers corresponding to the displacements marked in Fig. 11a. After the number 6 in Fig. 11b, the deformation is seen to proceed with asymmetric folding through 7-10. Totally, four folds form experimentally and numerically in double-end inward constraint tubes. In doubleend outward constraint tubes, the experiments and the model show almost the same load-displacement behavior and the number of folds (4) as shown in Fig. 12a. The numerical deformation history of double-end outward constraint tubes is further shown in Fig. 12b. The deformation sequence in these tubes shows very similar characteristics with those of free-constraint tubes, composing of the sequence of the inward and outward folding. In fully constraint tubes, two tubes crush in concertina mode, forming four folds, while one tube shows mixed mode of deformation (Fig. 13). The numerical deformation mode is, however, concertina as shown in Fig. 13. For comparison, the representative load-dis- placement curves of the double-end constraint tubes are shown in Fig. 14 together with that of the free-constraint tube. It is noted in Fig. 14 that, in double-end outward and fully constraint tubes, although the initial peak-load value is the same with that of the free-constraint tube, the initial drop-load is higher and almost equal to the following drop-load values. It is also noted that the intermediate part of the cycle is totally absent in experimentally tested outward constraint tubes. A similar effect of increased first drop-load is also seen in double-end fully constraint tubes deformed in concertina mode (Figs. 13 and 14).

In double-end inward-outward constraint tubes, two tubes deform experimentally in concertina with 4.5 folds (Fig. 15a), while one tube deformed in mixed mode, two axisymmetric folds followed by asymmetric mode of deformation (Fig. 15b). In inwardfully and outward-fully constraint tubes the deformation mode is concertina with 5 and 4.5 folds (Fig. 15c and d), respectively. In double-end inward-outward constraint tubes, the folding starts from the outward constraint end. While in inward-fully constraint tubes the folding starts from the fully constraint end and in outward-fully constraint tubes the folding starts from the fully constraint end in two tubes. The models used to simulate the tube deformation show essentially the same deformation modes and 


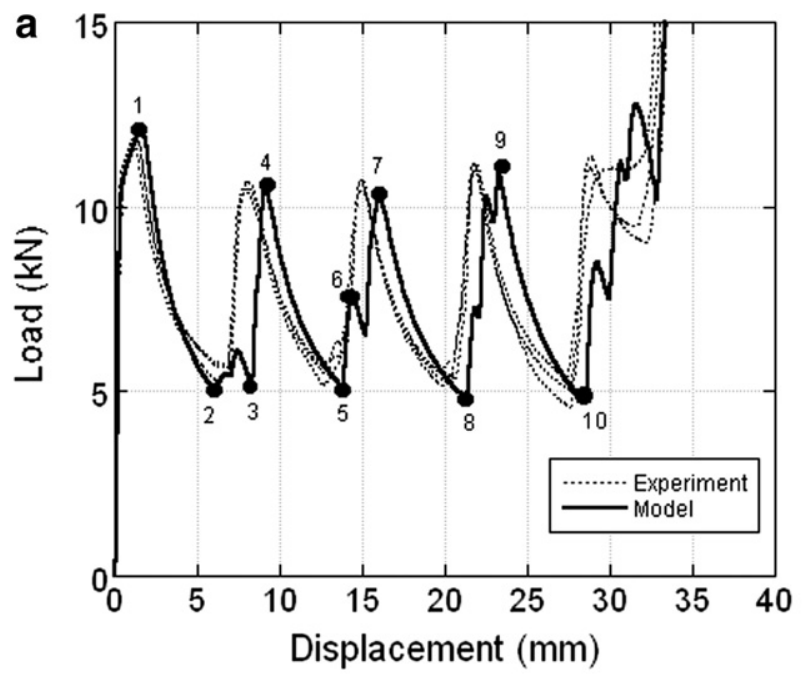

b
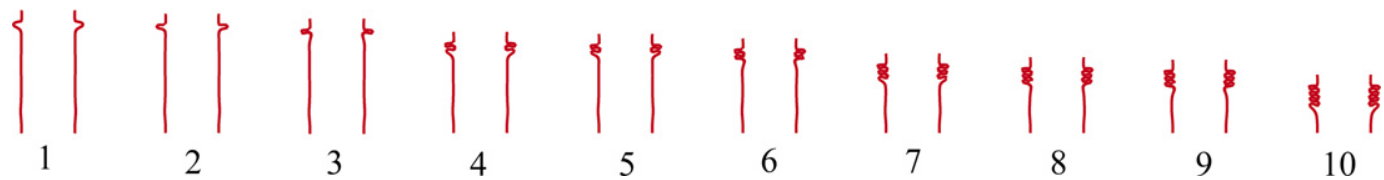

Fig. 12. (a) Experimental and numerical load-displacement curves and (b) numerical deformation history of double-end outward constraint tubes.

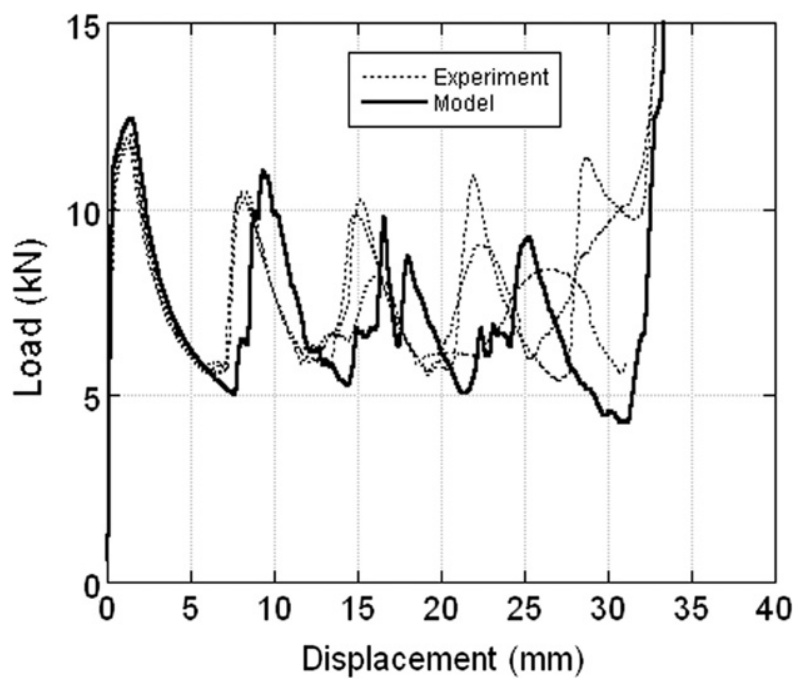

Fig. 13. Experimental and numerical load-displacement curves of double-end fully constraint tubes.

the numbers of folds as seen in Fig. 16. Fig. 17a and b show experimental and numerical representative load-displacement curves of the double-end inward-outward, inward-fully, outward-fully constraint and free-constraint tubes, respectively. In these constraint tubes, the experimental initial drop-load is higher than that of free-constraint tube as seen in Fig. 17a. The numerical load-displacement curves of the constraint tubes are essentially the same with that of free-constraint tube. In Fig. 18, experimental and numerical outward-fully constraint tube's load-displacement curves are shown for comparison. Experimental and modeling load-displacement curves show similar load values except the shift in displacements corresponding to the peak-loads. Generally, the modeling results in similar load values with those of experiments in double-end constraint tubes.

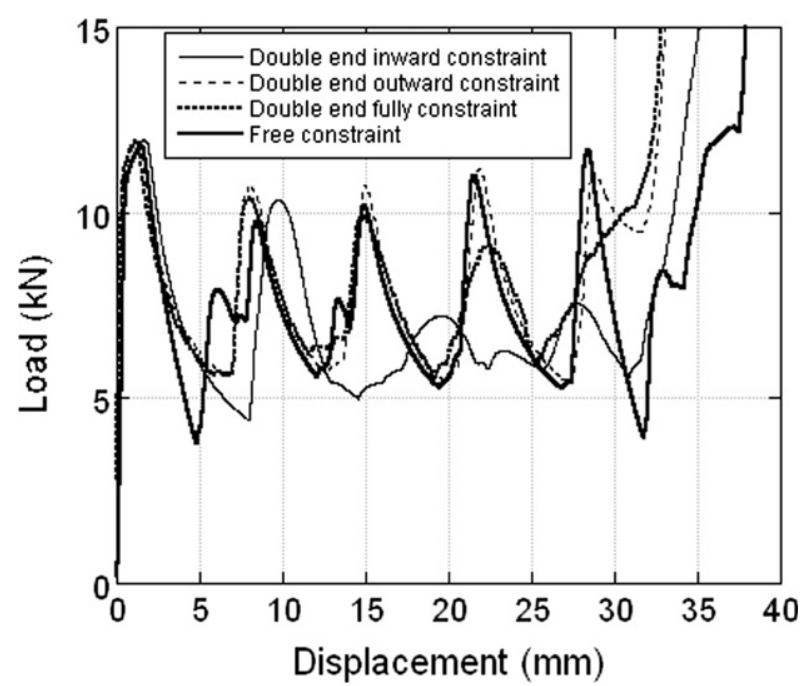

Fig. 14. Experimental load-displacement curves of double-end constraint tubes.

\section{Discussion}

The tubes tested in the present study, free and end-constraint tubes, $(D / t=22.2$ and $2.2 \leqslant L / D \leqslant 2.5)$ deformed dominantly in concertina mode of deformation, which is fully in accord with the previous studies $[8,9]$. However, few end-conditions were reverted the deformation mode into mixed/diamond mode of deformation, showing an effect of end constraining. Table 1 lists the fold starting ends and the deformation modes of experimentally and numerically tested free and end-constraint tubes. Since the folding always started and proceeded from the free-end, no constraint effect was found in single-end constraint tubes both in experiments and models. In double-end inward constraint tubes, the inward constraint end was reverted the deformation mode into mixed and/or diamond mode in both the experiments and the model. Dia- 


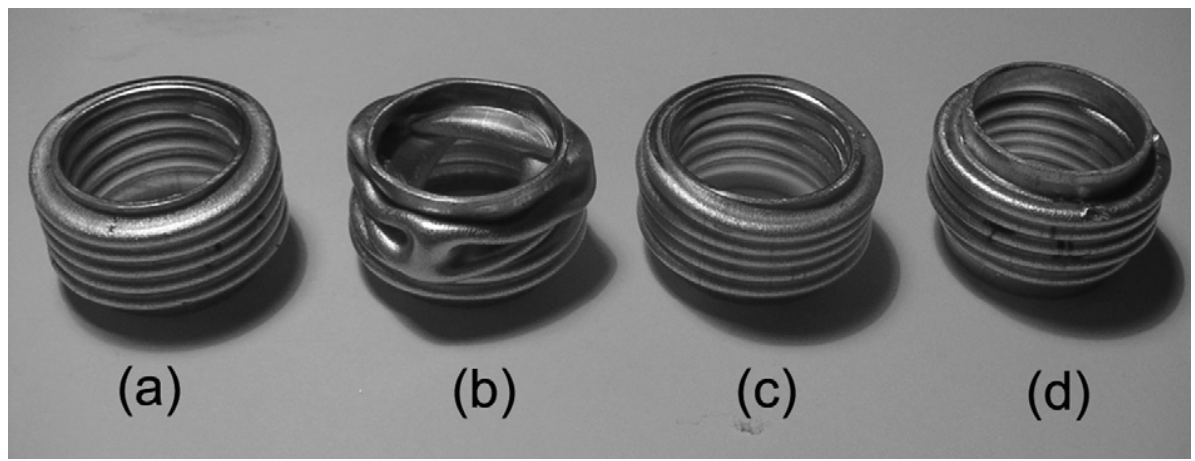

Fig. 15. Compressed double-end constraint tubes (a and b) inward-outward, (c) inward-fully and (d) outward-fully constraint.

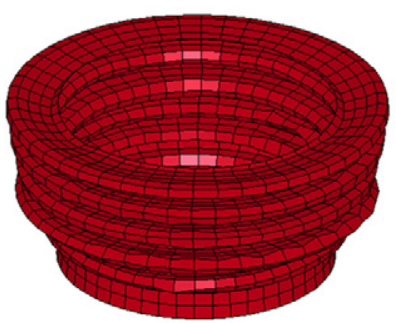

a

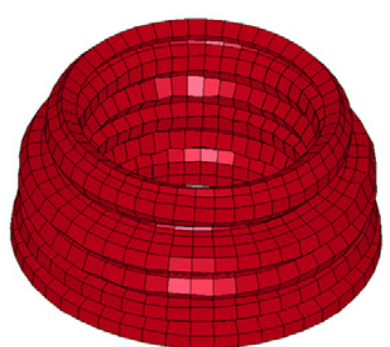

b

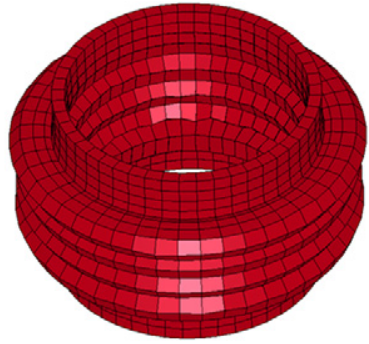

C

Fig. 16. Numerically compressed double-end constraint tubes (a) inward-outward, (c) inward-fully and (d) outward-fully constraint.
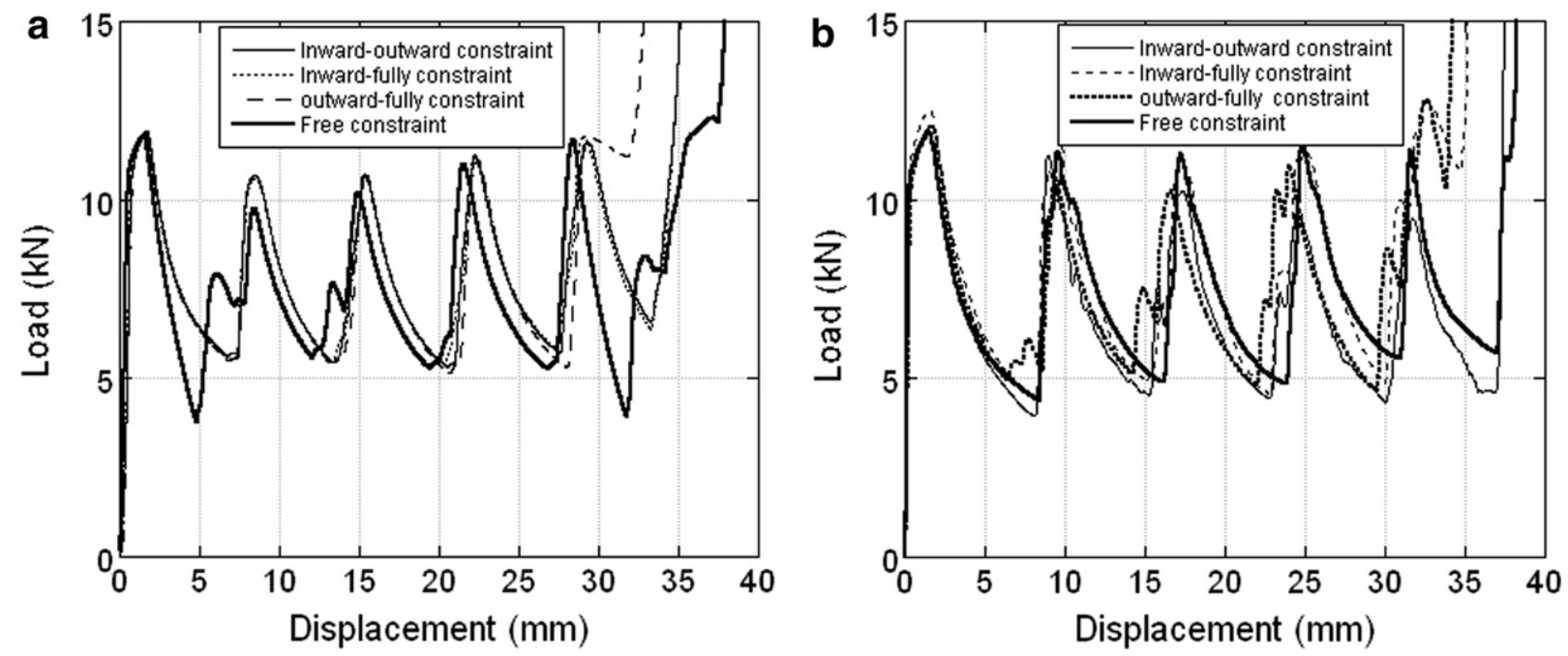

Fig. 17. Load-displacement curves of double inward-outward, inward-fully and outward-fully constraint tubes, (a) experiment and (b) model.

mond mode of deformation, being the lower deformation mode, results in a decrease in experimental and numerical average crushing loads particularly at increasing displacements as depicted in Fig. 19a and b, respectively. It is also noted in Fig. 19a that the fully and outward double-end constraining of the tube ends slightly increase the experimental average load values over those of freeconstraint tubes. The effect of end constraint on the initial peakload and the following initial drop-load values are shown in Fig. 20 for the studied constraint types. For free-end, single-end and double-end constraint tubes ( 1 through 10), the experimental and numerical initial peak-load and drop-load values generally show well agreements. An insignificant effect of end constraint is seen in numerical and experimental initial peak-load values, while the double-end constraining increases the drop-load values except the numerical drop-load values of double-end inward-outward and inward-outward constraint tubes. This effect is also seen in Fig. 17a in which the experimental initial peak-load values of double-end constraint tubes are about 20\% higher than those of empty tubes. The displacements corresponding to the initial peak-load are further shifted from $5 \mathrm{~mm}$ to $7.25 \mathrm{~mm}$ in double-end constraint tubes as seen in the same figure. This results in a slightly widened initial overshoot region and increases the average load values fol- 


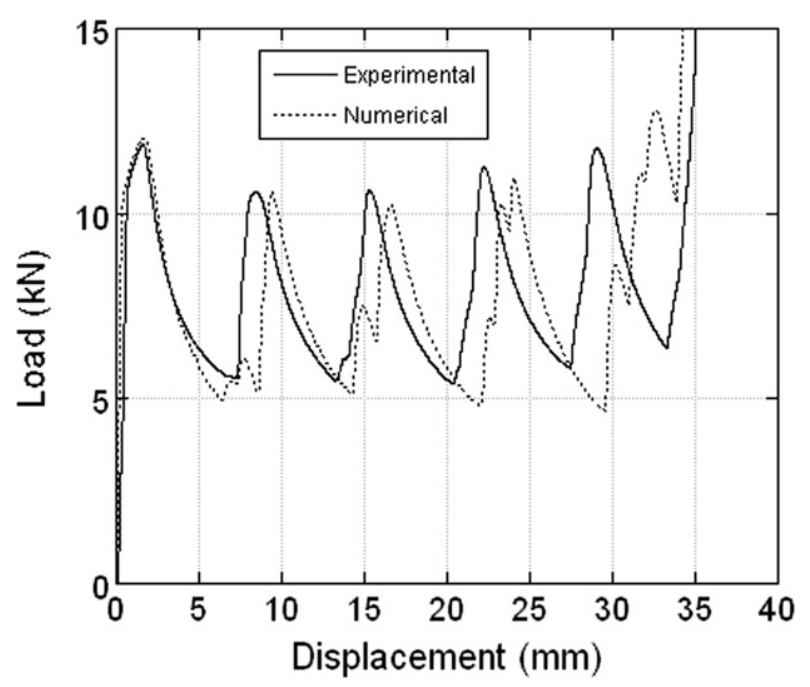

Fig. 18. Experimental and numerical load-displacement corves of double-end outward-fully constraint tube.

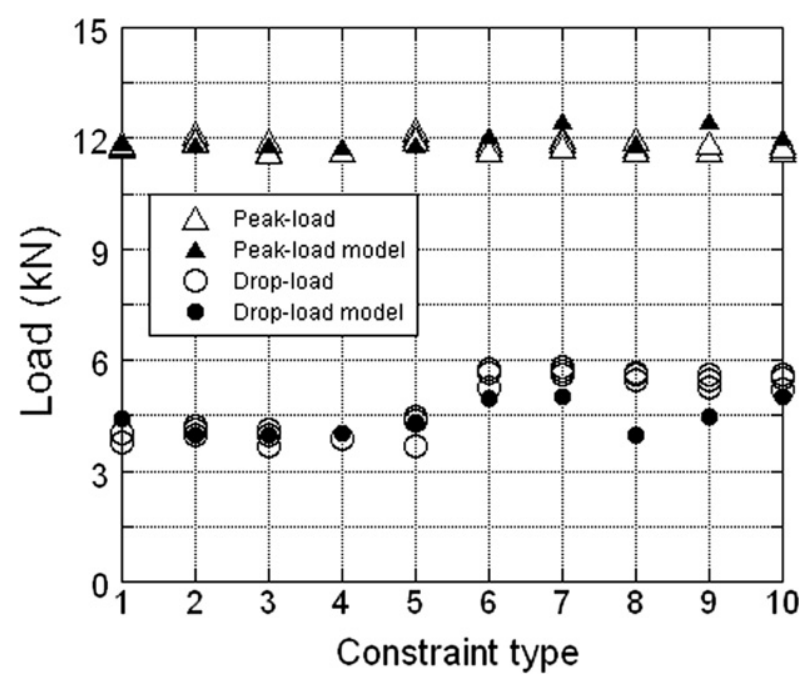

Fig. 20. Initial peak and drop-load values as function of constraint type.

Table 1

Deformation modes and fold starting ends of the tested tubes

\begin{tabular}{|c|c|c|c|c|}
\hline End-condition & $\begin{array}{l}\text { Experimental fold } \\
\text { starting end }\end{array}$ & $\begin{array}{l}\text { Numerical fold } \\
\text { starting end }\end{array}$ & Experimental mode of deformation & Numerical mode of deformation \\
\hline 1: Free & - & - & Concertina & Concertina \\
\hline $\begin{array}{l}\text { 2: Single-end inward } \\
\text { constraint }\end{array}$ & Free-end & Free-end & Concertina & Concertina \\
\hline $\begin{array}{l}\text { 3: single-end outward } \\
\text { constraint }\end{array}$ & Free-end & Free-end & Concertina & Concertina \\
\hline 4: Single-end fully constraint & Free-end & Free-end & Concertina & Concertina \\
\hline $\begin{array}{l}\text { 5: Double-end inward } \\
\text { constraint }\end{array}$ & - & - & $\begin{array}{l}\text { 2: Mixed (one axisymmetric fold and three } \\
\text { asymmetric folds), } 1 \text { : diamond }\end{array}$ & $\begin{array}{l}\text { Mixed (two axisymmetric folds and two } \\
\text { asymmetric folds) }\end{array}$ \\
\hline $\begin{array}{l}\text { 6: Double-end outward } \\
\text { constraint }\end{array}$ & - & - & Concertina & Concertina \\
\hline $\begin{array}{l}\text { 7: Double-end fully } \\
\text { constraint }\end{array}$ & - & - & 2: Concertina, $1:$ mixed ( $2-3$ axisymmetric folds) & Concertina \\
\hline $\begin{array}{l}\text { 8: Double-end inward- } \\
\text { outward constraint }\end{array}$ & Outward end & Outward end & 2: Concertina, $1:$ mixed ( 2 axisymmetric folds) & Concertina \\
\hline $\begin{array}{l}\text { 9: Double-end inward-fully } \\
\text { constraint }\end{array}$ & Fully end & Fully end & Concertina & Concertina \\
\hline $\begin{array}{l}\text { 10: Double-end outward- } \\
\text { fully constrain }\end{array}$ & Fully end & Fully end & Concertina & Concertina \\
\hline
\end{tabular}
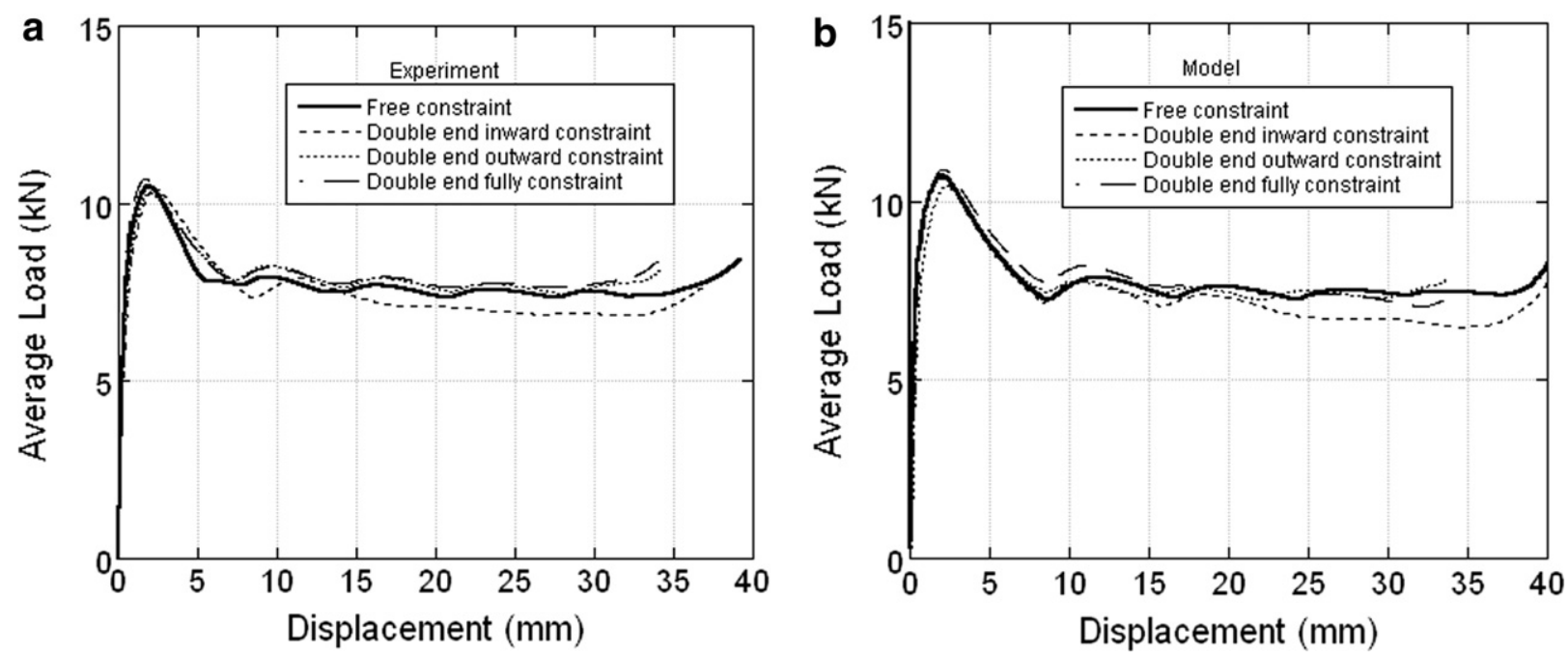

Fig. 19. Average load vs. displacement curves of double-end constraint tubes (5, 6 and 7): (a) experiment and (b) model. 

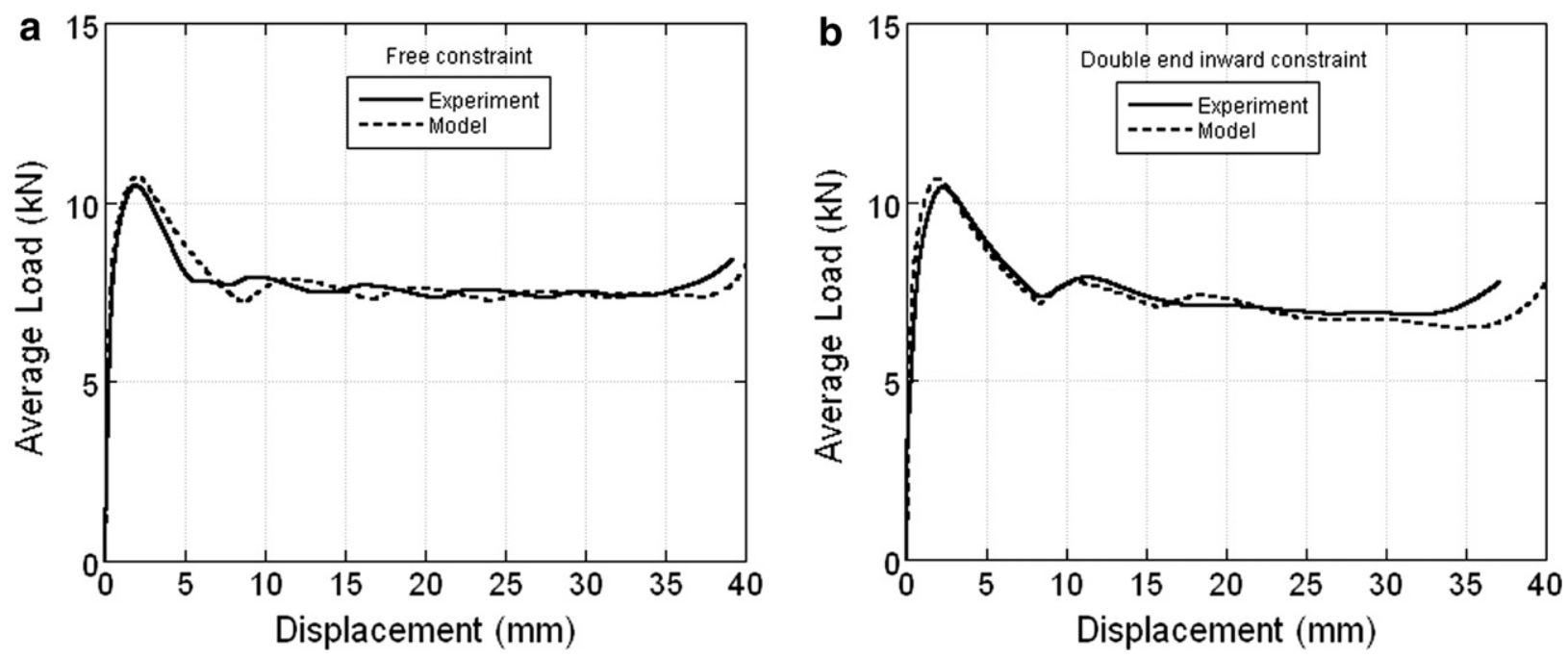

Fig. 21. Experimental and numerical average load vs. displacement curves of (a) free and (b) double-end inward constraint tubes.

lowing the initial overshoot region in experimental average loaddisplacement curves of double-end constraint tubes, except double-end inward constraint tubes, as compared with free-constraint tubes (Fig. 19a).

Fig. 21a and b show the experimental and numerical average load-displacement curves of free and double-end inward constraint tubes, respectively. In both constraint types the experimental and numerical average load values are very similar, showing the effectiveness of the modeling of tube deformation in predicting the average crushing load values in tubes with varying end-conditions. Due to the deformation mode transition from concertina to diamond and mixed modes, a 3D model was used in all simulations since as noted before, 2D models of tubes resulted in solely concertina mode of deformation, revealing no transition to diamond mode of deformation.

\section{Conclusion}

In this study the effect of end constraining on the deformation and load-displacement behavior of a 3003-H14 Al tube were experimentally and numerically investigated. Free and single-end constraint tubes deformed experimentally and numerically in concertina mode of deformation. In double-end constraint tubes of fully-inward constraint showed mixed/diamond mode of deformation mode experimentally and numerically, while double-end fully and inward-outward constraint tubes showed concertina/mixed mode of deformation experimentally. Double-end constraining of tube resulted in an increase in initial drop-load values, widening the initial overshot region in average load-displacement curves as compared with free-constraint tubes. Double-end inward constraint tubes further showed reductions in average load values particularly at increasing displacements. Well agreements found between numerical and experimental load and average load-displacement curves of the tubes with different end constraints showed that numerical tools could be used to predict the effect of end-constraining on the deformation mode and load-displacement behavior of tubes satisfactorily.

\section{Acknowledgement}

I thank Prof. Mustafa Guden for his valuable comments on the experimental and numerical results of the present study.

\section{References}

[1] Alexander JM. An approximate analysis of the collapse of thin cylindrical shells under axial loading. Q J Mech Appl Math 1960;13:10-5.

[2] Abramowicz W, Jones N. Dynamic axial crushing of circular tubes. Int J Impact Eng 1984;2:263-81.

[3] Abramowicz W, Jones N. Dynamic progressive buckling of circular and square tubes. Int J Impact Eng 1986;4:243-69.

[4] Wierzbicki T, Bhat SU, Abramowicz W, Brodkin D. Alexander revisited - a two folding elements model of progressive crushing of tubes. Int J Solids Struct 1992;29:3269-88.

[5] Gupta NK, Velmurugan R. Consideration of internal folding and non-symmetric fold formation in axisymmetric axial collapse of round tubes. Int J Solids Struct 1997;34:2611-30.

[6] Massimiliano A, Giovanni B, Antonio G, Roberto V. Crushing behaviour of aluminium square and rectangular tubes. Aluminium alloys: their physical and mechanical properties, pts 1-3, vol. 217. 1996. p. 1733-8.

[7] Singace AA. Axial crushing analysis of tubes deforming in the multi-lobe mode. Int J Mech Sci 1999;41:865-90.

[8] Singace AA, El-Sobky H. Uniaxial crushing of constrained tubes. Proc Int Mech Eng C - J Mech Eng Sci 2001;215:353-64

[9] Andrews KRF, England GL, Ghani E. Classification of the axial collapse of cylindrical-tubes under quasi-static loading. Int J Mech Sci 1983;25:687-96.

[10] Gupta NK, Abbas H. Mathematical modeling of axial crushing of cylindrical tubes. Thin-Walled Struct 2000;38:355-75.

[11] Hosseinipour SJ, Daneshi GH. Energy absorbtion and mean crushing load of thin-walled grooved tubes under axial compression. Thin-Walled Struct 2003;41:31-46.

[12] Han HP, Taheri F, Pegg N. Quasi-static and dynamic crushing behaviors of aluminum and steel tubes with a cutout. Thin-Walled Struct 2007;45:283-300.

[13] Seitzberger M, Rammerstorfer FG, Degischer HP, Gradinger R. Crushing of axially compressed steel tubes filled with aluminium foam. Acta Mech 1997; 125:93-105.

[14] Santosa SP, Wierzbicki T, Hanssen AG, Langseth M. Experimental and numerical studies of foam-filled sections. Int J Impact Eng 2000;24:509-34.

[15] Toksoy AK. Quasi-static axial compression behavior of empty and polystyrene foam filled aluminum tubes, MSc Thesis. Department of Mechanical Engineering, Izmir Institute of Technology, Izmir, Turkey, 2003.

[16] Guden M, Kavi H. Quasi-static axial compression behavior of constraint hexagonal and square-packed empty and aluminum foam-filled aluminum multi-tubes. Thin-Walled Struct 2006;44:739-50.

[17] Aljawi AAN. Numerical simulation of axial crushing of circular tubes. JKAU: Eng Sci 2002;14:101-15.

[18] Bardi FC, Yun HD, Kyriakides S. On the axisymmetric progressive crushing of circular tubes under axial compression. Int J Solids Struct 2003;40:3137-55.

[19] Al Galib D, Limam A. Experimental and numerical investigation of static and dynamic axial crushing of circular aluminum tubes. Thin-Walled Struct 2004:42:1103-37.

[20] Silcock M, Hall W, Fox B, Warrior N. Finite element modelling of metallic tubular crash structures with an explicit code. Int J Vehicle Safety 2006;1:292-303.

[21] Gupta NK. Some aspects of axial collapse of cylindrical thin-walled tubes. Thin-Walled Struct 1998;32:111-26. 